\title{
Gerencia educativa: comunicación interna en la Oficina General de Gestión de Recursos Humanos caso Perú
}

\author{
Educational management: internal communication in the General Office of Human Resources Management - \\ Peru case study
}

Gestão educacional: comunicação interna no Escritório Geral de Gestão de Recursos Humanos, Peru Estudo de caso

\author{
Aura Elisa Quiñones Li \\ auraelisa2006@hotmail.com \\ https://orcid.org/0000-0002-5105-1188 \\ Universidad César Vallejo, Lima-Perú \\ Delia María Macarlupu Guevara \\ deliamacarlupu@gmail.com \\ https://orcid.org/0000-0003-0259-4626 \\ Universidad César Vallejo, Lima-Perú
}

\author{
Luz Emerita Cervera Cajo \\ luzz-cervera@hotmail.com \\ https://orcid.org/0000-0003-1530-7761 \\ Universidad César Vallejo, Lima-Perú
}

César Augusto Quiñones Vernazza

cquinone1972@yahoo.es

https://orcid.org/0000-0002-5887-1795

Universidad César Vallejo, Lima-Perú

Artículo recibido 3 de septiembre 2021, arbitrado y aceptado 26 de octubre 2021 y publicado 28 de diciembre 2021

\section{RESUMEN}

La comunicación interna dentro de las organizaciones educativas denota debilidades en la actualidad producto de las fallas vías o estrategias para llevar a cabo este proceso. por lo que la intención del estudio es comparar la comunicación interna en trabajadores de la Oficina de Gestión de Recursos Humanos. Se trata de un estudio básico, el diseño es descriptivo comparativo. La población estuvo conformada por 139 empleados pertenecientes a la Oficina de Recursos Humanos de una Entidad Pública en Perú. Se utilizó como técnica la encuesta a fin de obtener información de los trabajadores, para evaluar cuál era su percepción respecto a la comunicación interna se utilizó la escala de percepción sobre la comunicación interna. En las mediciones efectuadas en trabajadores profesionales $(69,67)$ y técnicos $(64,86)$, se observa una mayor media en trabajadores profesionales; por lo que se concluye que existe diferencias en la percepción comunicación interna entre trabajadores profesionales y técnicos de la Oficina de Recursos Humanos.

Palabras clave: Comunicación interpersonal; Comunicación intrapersonal; Comunicación institucional

\section{ABSTRACT}

Internal communication within educational organizations currently shows weaknesses due to the lack of ways or strategies to carry out this process. Therefore, the purpose of the study is to compare internal communication in workers of the Human Resources Management Office. This is a basic study; the design is descriptive and comparative. The population consisted of 139 employees belonging to the Human Resources Office of a Public Entity in Peru. The survey was used as a technique to obtain information from the workers, to evaluate their perception regarding internal communication, the scale of perception on internal communication was used. In the measurements made on professional workers (69.67) and technical workers (64.86), a higher average is observed in professional workers; therefore, it is concluded that there are differences in the perception of internal communication between professional and technical workers of the Human Resources Office.

Key words: Interpersonal communication; Intrapersonal communication; Institutional communication

\section{RESUMO}

A comunicação interna dentro das organizações educacionais mostra atualmente pontos fracos devido à falta de meios ou estratégias para realizar este processo. Portanto, a intenção do estudo é comparar a comunicação interna nos funcionários do Escritório de Gestão de Recursos Humanos. Este é um estudo básico, o projeto é descritivo e comparativo. A população consistia em 139 funcionários pertencentes ao Escritório de Recursos Humanos de uma Entidade Pública no Peru. Uma pesquisa foi utilizada como técnica para obter informações dos trabalhadores, para avaliar sua percepção da comunicação interna, a escala de percepção da comunicação interna foi utilizada. Nas medições realizadas em trabalhadores profissionais $(69,67)$ e técnicos $(64,86)$, foi observada uma média mais alta em trabalhadores profissionais; portanto, conclui-se que existem diferenças na percepção da comunicação interna entre trabalhadores profissionais e técnicos no Escritório de Recursos Humanos.

Palavras-chave: Comunicação interpessoal; Comunicação intrapessoal; Comunicação institucional 


\section{INTRODUCCIÓN}

La gerencia educativa constituye uno de los principales pilares en la buena gestión de las instituciones académicas por ello, es relevante recoger un motor que hace que esto funcione dentro de las organizaciones educativas, como lo es la comunicación interna con el departamento de gestión o talento humano como ha bien se le conoce en la actualidad. Los procesos que se deben dar para que esta oficina funcione correctamente, $y$ de esta manera pueda garantizar la funcionalidad a gran escala, parte de tener una buena comunicación educativa. Debido a que, si esto ocurre la Educación en Perú da un paso enorme a la transformación de la educación de calidad a la que tanto se persigue en estos tiempos.

La pandemia ha demostrado los vacíos inmersos dentro del sector educativo comunicacional interno con el personal de las instituciones educativas, siendo un común denominador que afecta directamente a la población educativa. Por ende, es necesario el abordaje sobre cómo se vienen dado estos procesos en las organizaciones en Perú a fin de matizar y mejorar la comunicación interna.

Por lo que gestionar la comunicación interna, ha sido siempre un punto de inflexión tanto en las instituciones públicas y privadas, siendo que, en su mayoría, son en las instituciones públicas en donde se presenta una comunicación y clima organizacional deficiente. (Charry, 2018). Respecto a la comunicación interna, considerando que esta comunicación se da en todas las instituciones de manera formal o informal, resulta difícil para muchas organizaciones poder gestionarla de manera adecuada (Salazar, 2017).
La construcción del diálogo en un ambiente interprofesional permite la creación de una relación de integración entre los involucrados, lo que posibilita el reconocimiento del trabajo del otro y sus especificidades, esto es clave para lograr una comunicación efectiva entre profesionales, favoreciendo al ambiente laboral con una atención más segura y humanizada (Coifman et al., 2021). Es por esto por lo que las organizaciones deben contar con un soporte o modelo de gestión de sus comunicaciones, considerando el uso de las nuevas tecnologías, a fin de difundir su mensaje, mejorar el clima organizacional y dar paso a la mejora continua de la institución (Salazar, 2017).

En este sentido, se debe tener en cuenta el flujo de comunicación que se lleva dentro de una organización o institución, este puede ser vertical, horizontal, ascendente $\mathrm{o} / \mathrm{y}$ descendente (Costa, 2000). Por lo que se recomiendan que las instituciones cuenten con un canal de conducción que asegure que el mensaje llegue a cada destinatario, de esta forma evitar la comunicación de forma informal y que se generen rumores; por lo que la comunicación interna tiene que estar sistemáticamente organizada a fin de poder incluir la política institucional (Salazar, 2017).

A nivel local, en la Oficina de Recursos Humanos de un Ministerio en Perú a cargo de la educación presenta distorsiones en la comunicación entre los profesionales y/o técnicos, interfiriendo en su efectividad, identificando esto como un problema organizacional que no permite la eficaz comunicación, especialmente entre diferentes profesiones. 
La pregunta de investigación general plantea: ¿Cuál es la diferencia en la comunicación interna en trabajadores profesionales y técnicos de la Oficina General de Recursos Humanos de un Ministerio del Perú? Asimismo, se planteó cinco problemas específicos los cuales plantean ¿Cómo es la comunicación interna de trabajadores profesionales y técnicos de la Oficina General de Recursos Humanos?, por otro lado, se plantearon preguntas relacionadas con determinar ¿cuál es diferencia en la dimensión intrapersonal, interpersonal e institucional en trabajadores profesionales y técnicos de la Oficina General de Recursos Humanos de un Ministerio del Perú?

El objetivo general del estudio plantea la comparación en la comunicación interna de los trabajadores dentro de la Oficina General de Gestión de Recursos Humanos dentro de una entidad pública del Estado de Perú; asimismo , se plantearon objetivos específicos los cuales estuvieron relacionados a explicar y describir la comunicación interna tanto en trabajadores técnicos como profesionales de la Oficina General de Recursos Humanos; por otro lado, se plantearon también objetivos específicos basados en comparar las dimensiones interpersonal, intrapersonal e institucional en trabajadores estos trabajadores.

Este estudio sustenta y enfatiza la reflexión sobre las posturas de los trabajadores frente a la comunicación interna, permitiendo el debate, a fin de presentar una alternativa de solución respecto a los modelos de gestión existente. También detalla cómo se ha estado manejando la comunicación interna, proponiendo una nueva metodología que se ajusta a la realidad problemática de la oficina de talento humano y lo aplicable a las labores cotidianas. El estudio presenta herramientas metodológicas que se adaptan a las circunstancias de la oficina. Epistemológicamente, el estudio de comunicación interna permite interpretar las subjetividades que se presentan en el diálogo de la oficina.

\section{Sustento}

Bedoya (2017), realizó un estudio en donde destaca el flujo dentro de la comunicación ascendente y descendente la cual se direcciona mediante la figura de un coordinador. Además, considera que las relaciones de los compañeros deben prevalecer dentro de un clima de confianza que permita mantener el trabajo en equipo, el estímulo e integración, a fin de fortalecer los medios y canales comunicacionales para relacionar e impactar directamente entre la comunicada educativa.

En un estudio realizado por Salazar (2017) en Guayaquil, se confirma que la comunicación interna en las pymes del sector comercial es una herramienta estratégica eficaz para mantener un buen clima laboral, crecimiento y sostenibilidad de las organizaciones, sin importar el tamaño de éstas. Además, aporta ventajas competitivas como la flexibilidad, adaptación a nuevas tecnologías, respuesta positiva a cambios de mercado y la inclusión de la mano de obra.

Teodoro (2017) realizó una revisión de publicaciones relativas a la comunicación interna y el clima organizacional en organizaciones e instituciones sanitarias, el cual le permitió concluir que si la gestión se asocia al clima organizacional y la comunicación se logra la interacción, intercambio y diálogo entre los trabajadores de las instituciones, todo ello orientado y enfocado en los procesos que definen la organización de salud y el comportamiento de los trabajadores. 
López y Saboya (2017), asimismo señalan que la comunicación asertiva y operativa es la que más influye con un 55,8\%, la comunicación ascendente se encuentra en un nivel excelente con un $41,7 \%$, por el contrario, la comunicación descendente presenta mayores dificultades con el 17,3\% y el liderazgo transformacional también es deficiente en un $22,4 \%$.

Coifman et al., (2021) recolectaron datos a través del análisis de documentos, observación del participante y entrevistas semiestructuradas a 22 profesionales de la salud a fin de identificar los factores internos y externos que interfieren con la práctica comunicativa interprofesional de en una unidad de urgencias de un hospital general, encontrando que los profesionales reconocen que la comunicación es importante para la continuidad segura de la atención a los pacientes, pero las principales dificultados que identifican para una comunicación efectiva es la sobrecarga laboral, el hacinamiento, la ausencia de estándares de conductas, el déficit en la relación interprofesional y la poca experiencia de los profesionales, los cuales dificultan el intercambio de información para la toma de decisiones.

Rodríguez y Vázquez (2019) en su estudio realizado en España, investigan acerca de los cambios a través del tiempo de la comunicación organizacional, considerando esta como una herramienta que permite la inclusión y la mejora de muchos de los procesos de trabajo; por otro lado, resaltan la necesidad de los profesionales de incorporar nuevas capacidades que le permitan interrelacionarse con diferentes disciplinas. De este modo evalúan los impactos que ha tenido la tecnología, las influencias y la adaptación de las competencias de los profesionales.
Finalmente, Melo et al., (2019) realizaron una investigación cualitativa para analizar la relación entre las variables liderazgo, prácticas de gestión estratégica y continuidad en ocho ONGs (Organizaciones No Gubernamentales) de Pernambuco. Los datos fueron obtenidos mediante análisis de documentos, entrevistas semiestructuradas a los líderes de las ONGs y aplicación de cuestionarios a líderes y colaboradores; luego de su análisis, los autores concluyeron que existe una relación entre las variables liderazgo transformacional y las prácticas de gestión estratégica, así como el liderazgo transaccional está relacionado con aspectos internos, cumplimiento de normas, seguimiento de actividades, seguimiento de errores y desvíos del equipo; asimismo, el liderazgo carismático se caracterizó por una alta capacidad de comunicación entre los miembros del equipo.

Respecto a la teoría de la comunicación interna para Ricardo (2009) este proceso permite que las organizaciones y sus integrantes, recopilen información pertinente sobre la institución y los cambios o variantes que ocurren en esta, permitiendo que sus integrantes compartan esta información, favoreciendo de esta forma en el desarrollo de sus capacidades de cooperación y organización.

Por otro lado, Camacho (2010) enfatiza que las comunicaciones dentro de las organizaciones tienen que ser multidireccionales, donde el empleado pueda contar con un canal de comunicación que le permita acceder a la información pertinente y oportuna, además le posibilite expresar sus ideas e inquietudes.

Para Soares (2008) la comunicación es una dimensión de la interacción social, es decir, se basa y reproduce el significado de los recursos de un 
sistema social, pero siempre en contextos prácticos, por lo que no debe verse como un proceso centrado en la entrega de mensajes. En el ámbito de la comunicación organizacional, significa que solo podemos medir su efectividad si logramos, de alguna manera, captar la interpretación de los mensajes por parte del público objetivo.

Para Lemos (2011) la comunicación interna es un elemento de comunicación organizacional. Está dirigido a un público estratégico, fundamental para las organizaciones: sus empleados que, vinculados a la empresa a través de las relaciones laborales, inciden o se ven afectados por los procesos de comunicación interna en el cumplimiento de su misión y objetivos. Marchiori (2008, p. 213) afirma que: La comunicación interna [...]es una herramienta que fomenta el diálogo entre líderes y empleados. Brinda oportunidades para el intercambio de información a través de la comunicación, contribuyendo a la construcción del conocimiento, que se expresa en las actitudes de las personas [...].

Las dimensiones de la comunicación interna consideradas en el presente estudio son la comunicación interpersonal, intrapersonal e institucional. Respecto a la comunicación interpersonal, Sánchez (2008) indica que este tipo de comunicación se da en el interior del individuo, siendo que este interactúa en su mente consigo mismo. Respecto a la comunicación interpersonal, Wiemann (2011) manifiesta que estas se dan en contextos sociales y son importantes para la interrelación con otras personas, por otro lado, el autor Rodríguez (2008) refiere que con esta la información es posible catalogar la comunicación dentro de la organización como un deber y un derecho de los empleadores y empleados.

\section{MÉTODO}

El presente estudio tiene un enfoque cuantitativo, porque recolectó datos y los analizó numéricamente. (Hernández, Fernández y Baptista, 2014). El tipo de investigación fue básica, ya que según Hernández y Mendoza (2018) tiene la finalidad de describir, detectar y explicar las características de determinado problema. El diseño es descriptivo comparativo.

Para este estudio la población estuvo conformada por 139 empleados pertenecientes a la Oficina de Recursos Humanos del Ministerio de Educación. Se utilizó como instrumento la encuesta a fin de obtener información de los trabajadores, para evaluar cuál era su percepción respecto a la comunicación interna, se utilizó la escala de percepción sobre la comunicación interna, esta encuesta constó de 27 preguntas, fue de aplicación directa y tuvo una duración de 20 minutos, la escala fue de tipo Likert, se consideraron las dimensiones intrapersonal, interpersonal e institucional.

La validez del instrumento fue determinada por una prueba piloto y se utilizó la $\mathrm{R}$ de Pearson y el coeficiente alfa de Cronbach para la confiabilidad, a partir de aquí se eliminaron los ítems de la encuesta, que presentaban baja correlación, manteniendo 23 ítems que superaban el valor de 0. 20.Se analizaron los datos y se obtuvieron los valores a partir de frecuencias y porcentajes, prueba de normalidad Kolmogrov Smirnov, prueba de "t" de Student y la prueba "U" de Mann Whitney.

\section{RESULTADOS}

\section{Contrastación de hipótesis específica 1}

Los resultados obtenidos como promedio de la comunicación interpersonal en trabajadores 
profesionales y técnicos fueron de 25,96 y 25,39 respectivamente, notándose que los trabajadores profesionales poseen una mayor; sin embargo en la prueba " $U$ " de Mann Whitney, se comprueba que estas diferencias no son significativas $(p>, 05)$; por lo tanto, se acepta la hipótesis nula, es decir, no existe diferencias en la percepción comunicación intrapersonal entre trabajadores profesionales y técnicos de la Oficina de Recursos Humanos del Ministerio de Educación en Perú (Tabla 1).

Tabla 1. Comparación de la comunicación intrapersonal entre grupo de profesionales y técnicos.

\begin{tabular}{cccccc}
\hline Variable & Grupos & Media & “U” Mann Whitney & “Z” & P \\
\hline $\begin{array}{c}\text { Comunicación } \\
\text { intrapersonal } \\
\text { descendente }\end{array}$ & Personal Profesional & 25,96 & 2108,000 &,- 672 &, 502 \\
\hline
\end{tabular}

\section{Contrastación de hipótesis especifica 2}

Se obtuvieron resultados de 15,88 y 17,06 al comparar los promedios de comunicación interpersonal en los trabajadores profesionales y técnicos respectivamente, apreciando una mayor media en trabajadores técnicos; sin embargo, al emplear la prueba "t" de Student, se determinó que estas diferencias no son significativas ( $\mathrm{p}>$ ,05); es por esto que se acepta la hipótesis nula, no existe diferencias en la percepción comunicación interpersonal entre trabajadores profesionales y trabajadores técnicos de la Oficina de Recursos Humanos del Ministerio de Educación en Perú (Tabla 2).

Tabla 2. Comparaciones de comunicación interpersonal entre profesionales y técnicos.

\begin{tabular}{cccc}
\hline Variable & Grupos & Media & “t” \\
\hline Comunicación Interpersonal & Personal Profesional & 15,88 & $-1,915$ \\
& Personal Técnico & 17,06 \\
\hline
\end{tabular}

\section{Prueba de hipótesis especifica 3}

Los resultados respecto a la comunicación institucional presente en la Tabla 3, muestran una mayor media en los trabajadores profesionales $(27,83)$ a diferencia de los técnicos $(22,41)$; asimismo, tras realizar prueba " $U$ " de Mann Whitney, se rechaza la hipótesis nula, ya que la diferencia fue significativa $(\mathrm{p}<, 05)$; por lo que se concluye que respecto a la percepción en la comunicación institucional entre trabajadores profesionales $\mathrm{y}$ técnicos existe diferencias. 
Tabla 3. Comparación de la comunicación institucional entre trabajadores profesionales y técnicos.

\begin{tabular}{ccccc}
\hline Variable & Grupos & Media & “U” Mann Whitney & “Z” \\
\hline $\begin{array}{c}\text { Comunicación } \\
\text { Institucional }\end{array}$ & Personal Profesional & 27,83 & 993,000 & $-5,535$ \\
& & & & \\
& Personal Técnico & 22,41 & \\
\hline
\end{tabular}

\section{Contrastación de hipótesis general}

Se contrastó la hipótesis que plantea que existe diferencias en barreras de comunicación en el personal profesional y técnicos de la Oficina General de Recursos Humanos. Se obtuvieron resultado como resultado 69,67 y 64,86 , en los profesionales y técnicos respectivamente, observándose que los trabajadores profesionales poseen una mayor media; sin embargo, al emplear la prueba " $U$ " de Mann Whitney, se observa que estas diferencias no son significativas $(\mathrm{p}<, 05)$; por lo tanto, se rechaza la hipótesis nula y se acepta la alterna, es decir, existe diferencias en la percepción comunicación interna entre trabajadores profesionales y técnicos de la Oficina de Recursos Humanos. (Tabla 4).

Tabla 4. Comparación de la comunicación interna entre trabajadores profesionales y técnicos.

\begin{tabular}{|c|c|c|c|c|c|}
\hline Variable & Grupos & Media & “U” Mann Whitney & “Z” & $\mathbf{P}$ \\
\hline \multirow{2}{*}{$\begin{array}{l}\text { Comunicación } \\
\text { Institucional }\end{array}$} & Personal Profesional & 69,67 & 1809,000 & $-1,973$ & ,048 \\
\hline & Personal Técnico & 64,86 & & & \\
\hline
\end{tabular}

Respecto a la comunicación intrapersonal, se obtuvieron valores de 25.96 y 25.39 para los trabajadores profesionales y técnicos respectivamente, sin embargo, en la prueba de Mann Whitney, las diferencias no son significativas ( $\mathrm{p}>$ ,05). Por lo que se concluye que no hay diferencia en la dimensión intrapersonal en trabajadores profesionales y técnicos de la Oficina de Recursos Humanos del Ministerio de Educación en Perú.

Respecto a la comunicación interpersonal, se obtuvieron valores de 15.88 y 17.06 para los trabajadores profesionales y técnicos respectivamente, sin embargo, en la prueba de Mann Whitney, las diferencias no son significativas ( $\mathrm{p}>$ ,05). Por lo que se concluye que no hay diferencia en la dimensión interpersonal en trabajadores profesionales y técnicos de la Oficina de Recursos Humanos del Ministerio de Educación en Perú.

Respecto a la comunicación institucional, se obtuvieron valores de 27.83 y 22.41 para los trabajadores profesionales y técnicos respectivamente, así mismo, en la prueba de Mann 
Whitney, las diferencias son significativas ( $\mathrm{p}>$ ,05). Por lo que se concluye que existe diferencia en la dimensión institucional en trabajadores profesionales y técnicos de la Oficina de Recursos Humanos del Ministerio de Educación en Perú.

Respecto a la comunicación interna, se obtuvieron valores de 69.67 y 64.86 para los trabajadores profesionales y técnicos respectivamente, sin embargo, en la prueba de Mann Whitney, las diferencias no son significativas ( $p$ > ,05). Por lo que se concluye que no hay diferencia en la comunicación interna en trabajadores profesionales y técnicos de la Oficina de Recursos Humanos del Ministerio de Educación en Perú.

\section{Discusión}

Los resultados promedios de comunicación interna entre trabajadores profesionales y técnicos de la Oficina de Recursos Humanos del Ministerio de Educación es de 69,67 y 64,86 respectivamente, y de acuerdo a la prueba " $U$ " de Mann Whitney, estas diferencias no son significativas $(\mathrm{p}<, 05)$, es decir, la comunicación interna en ambos grupos de trabajadores se encuentra en el mismo nivel y no depende del grado de instrucción de los colaboradores, así como, no depende del tamaño de la institución u organización, según el estudio realizado por Salazar (2017), donde se resalta la importancia de la comunicación interna como herramienta estratégica eficaz para la generación de buenos climas laborales que propician el crecimiento y sostenibilidad de las organizaciones.

La relevancia de la comunicación interna, de acuerdo a Teodoro (2017) no solo se ve reflejada en el clima organizacional sino también en la gestión de las instituciones, la cual se logra con la interacción, intercambio y diálogo entre los trabajadores de las instituciones. Respecto a los promedios de la comunicación interpersonal, los resultados fueron de 15,88 y 17,06 para trabajadores profesionales y técnicos respectivamente, la diferencia entre dichos promedios es no significativa $(p>, 05)$; por lo tanto, no existe diferencias en la percepción de comunicación interpersonal, en ese sentido, Melo et al., (2019), reafirma la importancia del liderazgo carismático ya que tiene una influencia directa sobre la capacidad de comunicación entre los miembros del equipo de trabajo, del mismo modo, el liderazgo transaccional está relacionado con aspectos internos de la institución, el cumplimiento de normas, el seguimiento de actividades, errores y desvíos del equipo, todo éstas prácticas, refuerzan la comunicación interpersonal entre los colaboradores.

La comunicación institucional, presentó una media de 27,83 y 22,41 para los trabajadores profesionales y técnicos respectivamente, además de acuerdo a la prueba " $U$ " de Mann Whitney, si existe una diferencia significativa $(\mathrm{p}<, 05)$; en la percepción de la comunicación institucional entre ambos grupos de trabajadores, estos resultados son reforzados por el estudio realizado por Charry (2018), quién demostró que existe una correlación significativa entre la comunicación interna y las dimensiones grado de estructura y su influencia en el cargo ocupado por el trabajador; además demostró que existe una correlación significativa positiva y muy fuerte $(r=0.959)$ entre la comunicación interna y el clima de la organización. Asimismo, Monteiro et al. (2021), afirma que el proceso de comunicación organizacional interna está determinada por sus factores, los cuales deben ser brindados y reforzados 
por la organización, como, por ejemplo, garantizar el acceso y disponibilidad de las herramientas de comunicación interna.

\section{CONCLUSIONES}

Basado en el alcance que tuvo objetivo del estudio en relación a la comparación de la comunicación interna de los trabajadores dentro de la Oficina General de Gestión de Recursos Humanos dentro de una entidad pública del Estado peruano, se pudo evidenciar que no fueron significativa es decir, la comunicación interna en ambos grupos de trabajadores se encuentra en el mismo nivel y no depende del grado de instrucción de los colaboradores, así como, no depende del tamaño de la institución u organización, lo que da a interpretar que se debe trabajar dentro del sector público educativo en la mejora de la comunicación interna a fin de poder lograr cambios que se vean representados dentro de los procesos organizacionales y comunicacionales de cualquier institución educativa. c

\section{REFERENCIAS}

Bedoya Pastrana, S. M. (2017). Comunicación interna en instituciones educativas oficiales de la ciudad de Montería, Colombia: El Caso de la Institución Educativa Cristóbal Colón. In Del verbo al bit (pp. 1782-1794). Sociedad Latina de Comunicación Social

Camacho, A. (2010). Propuesta de un plan de comunicaciones internas orientado a la motivación de los empleados de la empresa Dinissan Santa Marta. (Tesis para optar el grado de maestría). Recuperado de: http:// www.usergioarboleda.edu.co/encontexto/ material/trabajos_de_grado/propuesta_plan_ comunicacion_motivacion_empleados_ dinnisan.pdf.
Charry, H. O. (2018). La gestión de la comunicación interna y el clima organizacional en el sector público.Comuni@cción-RevistadeInvestigación En Comunicación y Desarrollo, 9(1), 25-34-34

Coifman, A., Pedreira, L. , Jesus, A y Batista, R. E. A. (2021). Comunicação interprofissional em unidade de emergência: estudo de caso. Revista Da Escola de Enfermagem Da USP, 55, 1-8. https://doi.org/10.1590/s1980220x2020047303781

Costa, J (2000). Comunicación empresarial: Nuevas tendencias en comunicación para potenciar la estrategia empresarial. España, Barcelona

Hernández R., Fernández C. y Baptista P. (2014) Metodología de la investigación. México, impreso por Edamsa Impresiones, S.A. de C.V

Hernández, R. y Mendoza, C (2018). Metodología de la investigación. Las rutas cuantitativa, cualitativa y mixta, Ciudad de México, México: Editorial Mc Graw Hill Education, Año de edición: 2018, ISBN: 978-1-4562-6096-5, 714 p

Lemos, E (2011). La comunicación interna como diferencial en las relaciones públicas. En: FARIAS, Luiz Alberto de (org.). Relaciones públicas estratégicas - Técnicas, conceptos e instrumentos. São Paulo

López, M., y Saboya, N. (2017). Influencia de la comunicación interna en el nivel de clima organizacional en la Municipalidad de Chaclacayo. Revista de Investigación Universitaria, 5(1), 44-51. https://doi. org/10.17162/riu.v5i1.859

Marchiori, M. (2008). La comunicación interna: factor estratégico para el éxito empresarial. Rostros de la cultura organizacional y la comunicación. 2 ed São Caetano do Sul: Difusión, p.207- 224

Melo A., Borba, M., Correia, M. I. S., y Cabral, R. M. (2019). Liderança e gestão: um estudo em Organizações Não Governamentais (ONGs) na região metropolitana do Recife, PE. Interações (Campo Grande), 1149-1164. https://doi. org/10.20435/inter.v20i4.2077 
Monteiro, C., Kuhl, M. R., y Angnes, J. S. (2021). The process of internal organizational communication: A study done on a commercial and business managment association of Paraná state. In Perspectivas em Ciencia da Informacao (Vol. 26, Issue 1). https://doi.org/10.1590/1981$5344 / 3975$

Ricardo, C (2009). Comunicación organizacional: ¿estrategias exitosas? En: CONGRESO LUSOFÓNICO DE CIENCIAS DE LA COMUNICACIÓN, 7, 2009, Lisboa. Procedimientos electrónicos

Rodríguez, V. (2008). Comunicación corporativa: Un derecho y un deber. Santiago: Editores.

Rodríguez, L. y Vázquez, P. (2019). "Retos y perspectivas en la comunicación organizacional". El profesional de la información, v. 28, n. 5, e280501. https://doi.org/10.3145/epi.2019. sep. 01

Sánchez, J. (2008).Comunicación y construcción de conocimiento en el nuevo espacio tecnológico. Revista de Universidad y sociedad del conocimiento. Recuperado de http://www. uoc.edu/rusc/5/2/dt/esp/conocimiento_ tecnologico.pdf

Salazar Merchán, A. M. (2017). La comunicación interna como desafío en las PyMES comerciales de Guayaquil. INNOVA Research Journal, 2(10.1), 165-174. https://doi.org/10.33890/ innova.v2.n10.1.2017.499

Soares, A (2008). Comunicación y organizaciones bajo la influencia de la complejidad y el riesgo: un análisis conceptual. En: Eduardo de Jesús; Mozahir Solomon. (Org.).Interacciones plurales: comunicación y lo contemporáneo

Teodoro, M. (2017). La Comunicación interna y el clima organizacional en la gestión de las instituciones sanitarias. Dialnet. Dialnet, 5(1), 39-44. https://dialnet.unirioja.es/servlet/ articulo? codigo $=6570435$

Wiemann, M. (2011). La comunicación en las relaciones interpersonales. España.: UOC (Universitat Oberta de Catalunya) 